\title{
Multiparameter analysis of homogeneously R-CHOP-treated diffuse large B cell lymphomas identifies CD5 and FOXP1 as relevant prognostic biomarkers: report of the prospective SAKK 38/07 study
}

\author{
Alexandar Tzankov ${ }^{1 *}$, Nora Leu', Simone Muenst ${ }^{1}$, Darius Juskevicius ${ }^{1}$, Dirk Klingbiel ${ }^{2}$, Christoph Mamot ${ }^{3}$ \\ and Stephan Dirnhofer ${ }^{1}$
}

\begin{abstract}
Background: The prognostic role of tumor-related parameters in diffuse large B cell lymphoma (DLBCL) is a matter of controversy.

Methods: We investigated the prognostic value of phenotypic and genotypic profiles in DLBCL in clinical trial (NCT00544219) patients homogenously treated with six cycles of rituximab, cyclophosphamide, hydroxydaunorubicin, vincristine, prednisone (R-CHOP), followed by two cycles of R (R-CHOP-14). The primary endpoint was event-free survival at 2 years (EFS). Secondary endpoints were progression-free (PFS) and overall survival (OS). Immunohistochemical (bcl2, bcl6, CD5, CD10, CD20, CD95, CD168, cyclin E, FOXP1, GCET, Ki-67, LMO2, MUM1p, pSTAT3) and in situ hybridization analyses (BCL2 break apart probe, C-MYC break apart probe and C-MYC/IGH double-fusion probe, and Epstein-Barr virus probe) were performed and correlated with the endpoints.
\end{abstract}

Results: One hundred twenty-three patients (median age 58 years) were evaluable. Immunohistochemical assessment succeeded in all cases. Fluorescence in situ hybridization was successful in 82 instances. According to the Tally algorithm, 81 cases (66 \%) were classified as non-germinal center (GC) DLBCL, while 42 cases (34 \%) were GC DLBCL. BCL2 gene breaks were observed in 7/82 cases (9\%) and C-MYC breaks in 6/82 cases (8\%). "Double-hit" cases with BCL2 and C-MYC rearrangements were not observed. Within the median follow-up of 53 months, there were 51 events, including 16 lethal events and 12 relapses. Factors able to predict worse EFS in univariable models were failure to achieve response according to international criteria, failure to achieve positron emission tomography response $(p<0.005)$, expression of CD5 ( $p=0.02)$, and higher stage $(p=0.021)$. Factors predicting inferior PFS were failure to achieve response according to international criteria $(p<0.005)$, higher stage $(p=0.005)$, higher International Prognostic Index (IPI; $p=0.006)$, and presence of either C-MYC or BCL2 gene rearrangements $(p=0.033)$. Factors predicting inferior OS were failure to achieve response according to international criteria and expression of FOXP1 $(p<0.005)$, cyclin $\mathrm{E}$, CD5, bcl2, CD95, and pSTAT3 ( $p=0.005,0.007,0.016$, and 0.025, respectively). Multivariable analyses revealed that expression of CD5 $(p=0.044)$ and FOXP1 $(p=0.004)$ are independent prognostic factors for EFS and OS, respectively.

Conclusion: Phenotypic studies with carefully selected biomarkers like CD5 and FOXP1 are able to prognosticate DLBCL course at diagnosis, independent of stage and IPI and independent of response to R-CHOP.

Keywords: DLBCL, Prognosis, Phenotype, FISH, Prospective trial, CD5, FOXP1

\footnotetext{
*Correspondence: alexandar.tzankov@usb.ch

'Institute of Pathology, University Hospital Basel, Schoenbeinstrasse 40, CH-4031 Basel, Switzerland

Full list of author information is available at the end of the article
} 


\section{Background}

Diffuse large B cell lymphoma (DLBCL) is the most common nodal lymphoid malignancy, comprising approximately $30 \%$ of all adult lymphomas, with a rapidly rising incidence $[1,2]$. DLBCL demonstrates an aggressive clinical course, but potentially $60-70 \%$ of patients can be cured with the established rituximab, cyclophosphamide, hydroxydaunorubicin, vincristine, prednisone (R-CHOP) treatment standard [3]. Prediction of survival and stratification of patients for risk-adjusted therapy is based on the International Prognostic Index (IPI) [4]. R$\mathrm{CHOP}$ has not only led to a marked improvement of survival in DLBCL but has also called into question the significance of the IPI [5], leading to introduction of the revised IPI (R-IPI) [6]. Recent data suggests that IPI and R-IPI no longer reliably identify DLBCL risk groups with a $<50 \%$ chance of survival, despite about $30-40 \%$ of patients will still die of/with disease. Thus, there is a need for additional, particularly tumor-related, prognostic (and predictive) factors in DLBCL [7].

To date, only a limited number of tumor-related prognostic parameters exist for DLBCL like presence of $C$ $M Y C$ rearrangements or co-expression of bcl2 and c-myc. The morphological heterogeneity of DLBCL is reflected by significant molecular diversity at the genotypic, gene expression, and phenotypic levels [8,9]. Gene expression profiling data convincingly showed that DLBCLs are derived from germinal center $B$ cells (GCB) or activated $B$ cells $(\mathrm{ABC})$ [9-11]. Although the scientific evidence is robust and prognostically relevant, its translation into daily practice remains impractical because of the required high standard of tissue preservation, procedure duration, and costs. This problem prompted the search for molecular prognostic markers applicable to routine biopsies from patients with DLBCL. As a result, a large body of surrogate (phenotypic) models and algorithms to identify GCB and non-GCB DLBCL have been proposed and linked to outcomes [12]. Unfortunately, reliability and reproducibility of these models is often poor, impeding their translation into standard practice to predict survival and stratify patients for risk-adjusted therapy [12-14]. Technical issues, poor study designs, lack of standardization of evaluation procedures, and, particularly, lack of prospective trials all prevent an efficient clinical translation. A PubMed search for "DLBCL," "R-CHOP," "prognostic," "marker," and "prospective" identifies only a few prospective studies, in which biomarkers have been considered (e.g., [15-24]). Thus, there is an unmet requirement for further marker validation in prospective trials.

The translational study of the clinical trial "SAKK 38/ 07 Prospective evaluation of the prognostic value of positron emission tomography (PET) in patients with diffuse large B-cell-lymphoma under R-CHOP-14. A multicenter study" offered a unique opportunity to prospectively analyze the prognostic and predictive value of phenotypic and genotypic biomarkers suggested to play a prognostic role in DLBCL on a well-documented and homogenously treated clinical trial collective.

\section{Materials and methods}

\section{Patient recruitment, selection, and treatment}

The recruitment of patients for the SAKK 38/07 study started in November 2007 and finished in June 2010. Evaluation of the prognostic value of metabolic responses, as assessed by early PET after two cycles of R-CHOP-14, to identify a poor outcome patient subgroup was the main objective. PET was performed before, after two cycles of therapy, and at the end of treatment and was evaluated according to a 5-point scoring system with a cutoff determining positivity being set at 4 points (moderately increased uptake compared with the liver) [25]. The primary endpoint was event-free survival (EFS) at 2 years, and the secondary endpoints were progression-free (PFS) and overall survival (OS) after 2 and 5 years as well as the objective responses according to international criteria [26]. In accordance with the statistical advice for reaching sufficient power to address the two endpoints, recruitment of 154 patients was aimed. Because of concurrent registrations on the last recruitment day, 156 instead of 154 patients were recruited. Inclusion criteria were histologically proven diagnosis of CD20-positive DLBCL (no pretreatment revision of the slides by an expert hematopathologist was planned) including all Ann Arbor stages, tumor size $>14 \mathrm{~mm}$ on CT or MRI (because lymph nodes $\geq 15 \mathrm{~mm}$ are considered "pathologic" on computerized imaging), PET positivity of the tumors (documented 2 weeks to 4 days prior to registration), performance status $0-2$ on the ECOG scale, age $>17$, as well as no evidence of symptomatic central nervous system (CNS) disease, HIV, and/or hepatitis infection [27]. The study treatment consisted of R-CHOP given for six cycles followed by additional two applications of rituximab every 2 weeks (R-CHOP-14). Additionally, G-CSF support was given. The patients were asked to provide informed consent for the study and, separately, for the translational research. The primary pathology institutions were asked to send representative paraffin blocks for translational research after accomplishing the in-house diagnostic procedures to the Institute of Pathology at the University Hospital Basel. The study was approved by the Ethics Committee Beider Basel. Details of the SAKK 38/07 study are reported elsewhere [28].

\section{In situ biomarker analysis}

Immunohistochemical (bcl2, bcl6, c-myc, CD5, CD10, CD95, CD168, cyclin E, FOXP1, GCET, LMO2, MUM1p, pSTAT3) and in situ hybridization analyses [BCL2 
break apart probe (BAP), C-MYC BAP and C-MYC/IGH double-fusion probe (DFP), and Epstein-Barr virus probe (EBER)] were performed and correlated with clinicopathological parameters and clinical endpoints. Cell of origin (COO) was determined according to the Tally algorithm [29]. Additionally, selected cases were stained for CD23, CD30, cyclin D1, D2, D3, Ki-67, p27, p63, and SOX11 for specification of diagnosis. Reagent sources, pretreatment and incubation conditions, and cutoff scores are listed in Table 1. Immunohistochemical markers were assessed by microscopic counting of positive cells/tumor cells and were recorded in $5 \%$ increments in the primary statistical table. All cases were scored after training by at least two observers (either AT, SM, or SD), and only markers for which Cronbach's alpha analysis suggested good agreement between observers (alpha $>0.75$ ) were considered for prognostic evaluation. Relevant cutoff scores were either taken from the literature $[29,30]$ or calculated applying receiver operating characteristic (ROC) analysis [12]. Discrepancies in the results for evaluated markers, which were almost exclusively due to differential assessment of weak staining signals, were discussed at a double-headed microscope and the concordant result was considered. Fluorescence in situ hybridization (FISH) was performed exactly as described elsewhere [31]. All cases were FISH-scored twice (NL and $\mathrm{AT}$ ) with an excellent agreement $(\mathrm{alpha}=1)$ between both observers.

\section{Statistics}

All statistical analyses were performed using the Statistical Package of Social Sciences (IBM SPSS version 19.0, Chicago, IL, USA) for Windows and reported applying the REMARK guidelines [32]. The inter-observer agreement was assessed using the Cronbach's alpha reliability analysis; an alpha value of $>0.75$ indicates very good agreement. The Spearman rank correlation was used to analyze relationships between biomarkers and clinical and laboratory parameters; only correlations with a rho $\geq \pm 0.300$ were considered. The Mann-Whitney $U$ and Kruskal-Wallis tests were applied, where appropriate, to identify quantitative differences between groups. The prognostic performance of variables and determination of optimal cutoff values (except those extracted from the most recent literature) was assessed by ROC curve plotting sensitivity versus 1 -specificity with special consideration of the respective area under the ROC (AUROC). The optimal cutoff point was calculated using Youden's index $(Y)$, denoting $Y=$ sensitivity + specificity -1 , since this method can be applied to find the optimal unbiased cutoff value with the highest sensitivity and specificity [12]. OS was measured from registration to death or last follow-up, PFS from registration to relapse, death of any cause, or to last follow-up, and EFS from registration to relapse or death of any cause, initiation of any nonprotocol anticancer treatment because of lymphoma symptoms or need of concomitant radiotherapy or to

Table 1 Applied biomarker panel

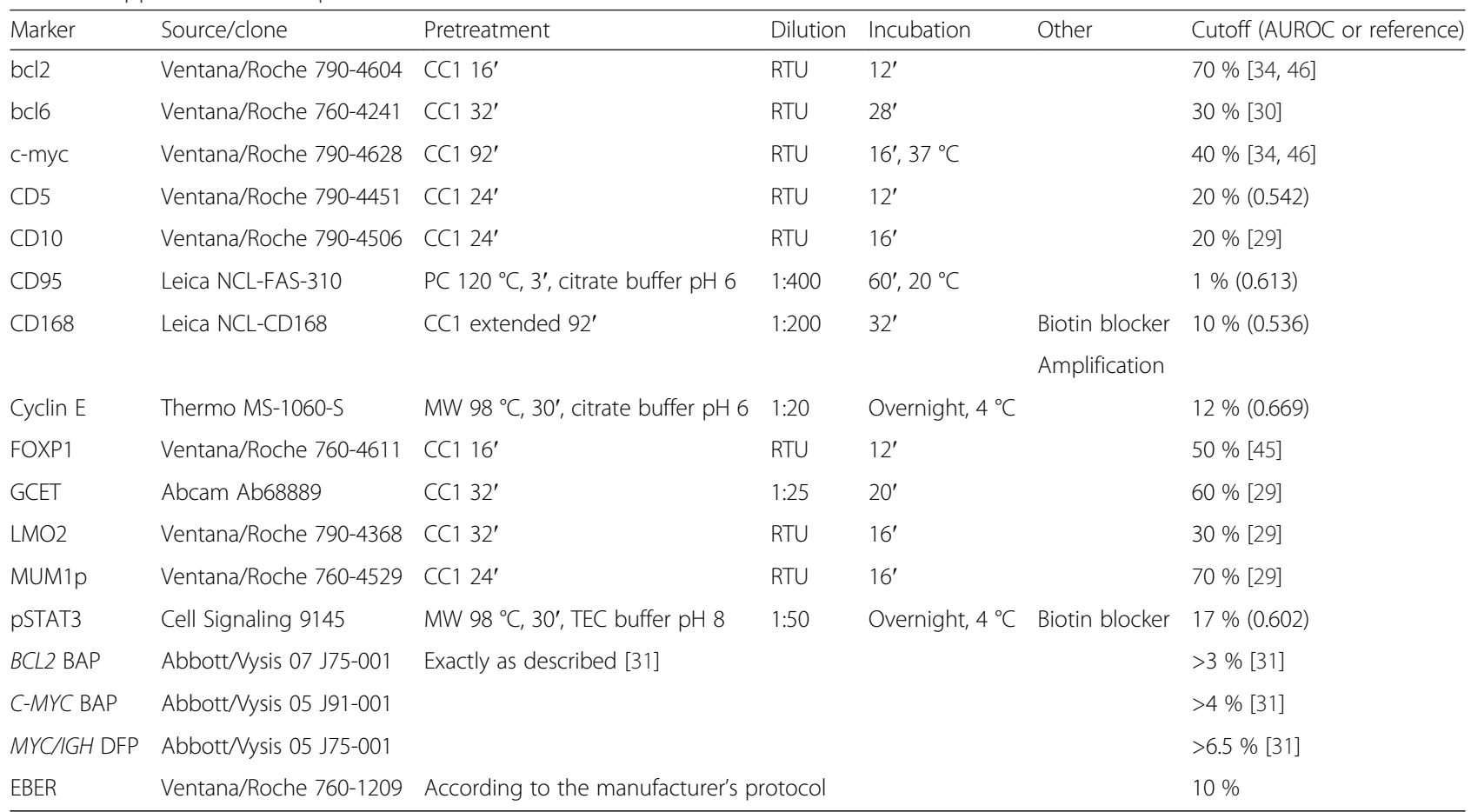

For diagnostic purposes and to "subtract" CD3-positive T cells in CD5-positive DLBCL, CD3 and CD20 stainings were also performed, but these were not considered biomarkers sensu stricto 
last follow-up. The probabilities of survival were determined using the Kaplan-Meier method, and differences were compared using the log-rank test. All biomarkers of prognostic significance in univariable models underwent multivariable analysis using the Cox proportional hazards model in a two-step manner since only that response criterion (either according to international criteria or PET or combined PET/CT response) with the highest relevance in an independent first step Cox model, run without biomarkers, was considered and compared to the biomarkers in the second step. All $p$ values were two-sided and considered statistically significant if $<0.05$. No adjustment for multiple testing was applied for secondary analyses because they were considered hypothesis generating and exploratory.

\section{Results}

Patients, case review, and clinico-pathologic characteristics

Nineteen patients refused a participation in the translational research part of the project. In 11 cases, no material for translational research was present. Thus, 126 cases were further studied: DLBCL diagnosis could not be confirmed in three of these cases by conventional morphology and additional immunohistochemical evaluation (the final diagnosis of marginal zone lymphoma was established in two cases and one turned to be a blastoid mantle cell lymphoma). Thus, the analysis was finally performed on 123 cases. Patient characteristics are given in Table 2. Survival data were complete for 116 patients.

Eighty-nine lymphomas were primary nodal or of lymphoid tissue (including the mediastinum, the spleen, and Waldeyer's ring), while 34 were extranodal (most commonly soft tissue, gastrointestinal tract, and bones). Based on integrative analysis, 100 cases were shown to be centroblastic DLBCL, five were immunoblastic DLBCL, three were anaplastic DLBCL, six were unclassifiable, six were primary mediastinal large B cell lymphomas (PMBL; thereof, two were nodal DLBCL with morphologic and phenotypic features of PMBL), two were $\mathrm{T}$ cell- and histiocyte-rich B cell lymphomas (THRBCL), and one was a lymphomatoid granulomatosis (LG) grade 3.

The study material consisted of 66 (54 \%) lymphadenectomy specimens that were studied on tissue microarrays (TMA) and 57 (46\%) cases with only small core needle biopsy material available, which were considered non-arrayable and were studied on conventional serial sections. Arrayable cases were brought into a TMA format applying the 1-mm core needle as described [33].

\section{In situ biomarkers}

Immunohistochemistry was evaluable in all cases, while FISH was successful in $82(67 \%)$ instances (Table 3, Fig. 1a-d); importantly, cases in which FISH failed were evenly distributed among arrayable lymphadenectomy
Table 2 Basic patient characteristics

\begin{tabular}{|c|c|c|}
\hline Age, median (range) & & $58(18-81)$ \\
\hline \multirow[t]{2}{*}{ Gender, N (\%) } & $\mathrm{F}$ & $68(55)$ \\
\hline & M & $55(45)$ \\
\hline \multirow[t]{5}{*}{ Stage, $N(\%)$} & । & $12(10)$ \\
\hline & $\|$ & $41(34)$ \\
\hline & III & $30(24)$ \\
\hline & IV & $39(32)$ \\
\hline & Missing & 1 \\
\hline \multirow[t]{6}{*}{$\mid \mathrm{PI}, \mathrm{N}(\%)$} & 0 & $23(19)$ \\
\hline & 1 & $36(29)$ \\
\hline & 2 & $27(22)$ \\
\hline & 3 & $20(16)$ \\
\hline & 4 & $13(11)$ \\
\hline & 5 & $4(3)$ \\
\hline \multirow{5}{*}{$\begin{array}{l}\text { Treatment response according } \\
\text { to international criteria, } N(\%)\end{array}$} & $C R$ & $102(83)$ \\
\hline & $P R$ & $18(15)$ \\
\hline & SD & $2(2)$ \\
\hline & PD & $0(0)$ \\
\hline & Missing & 1 \\
\hline \multirow[t]{4}{*}{$\begin{array}{l}\text { Combined metabolic and } \\
\text { morphologic responses, } N(\%)\end{array}$} & $\begin{array}{l}\text { Complete metabolic and } \\
\text { morphologic response }\end{array}$ & $68(59)$ \\
\hline & $\begin{array}{l}\text { Complete metabolic } \\
\text { response with residual mass }\end{array}$ & $24(21)$ \\
\hline & $\begin{array}{l}\text { Partial metabolic response } \\
\text { with residual mass }\end{array}$ & $23(20)$ \\
\hline & Missing & 8 \\
\hline \multirow[t]{2}{*}{ Collecting institutions, N (\%) } & University hospitals & $33(27)$ \\
\hline & Other hospitals & $90(73)$ \\
\hline
\end{tabular}

specimens and small core needle biopsy specimens but were more commonly observed in tissues from certain primary pathology institutions. Taking into consideration the Tally algorithm, 81 cases (66\%) were classified as non-GCB DLBCL, while 42 cases (34 \%) were GCB DLBCL; after excluding the PMBL, THRBCL, and LG, there were $39 \mathrm{GCB}$ and 75 non-GCB cases. BCL2 gene breaks were observed in $7 / 82$ cases $(9 \%) ; 6$ of the 7 (86\%) rearranged cases were of the GCB type. Two cases (all of the non-GCB type) showed BCL2 amplifications. $C-M Y C$ breaks were observed in $6 / 82$ cases $(8 \%)$; 4 were of the GCB type. Of the C-MYC rearranged cases, only 2 displayed $C-M Y C / I G H$ fusions, detectable by both DFP and BAP and corresponding to $t(8 ; 14)$, while $C-M Y C$ rearrangements were detectable only by $\mathrm{BAP}$ in the other 4 cases and were thus assumed to have occurred with alternative non-IGH C-MYC rearrangement partners. "Genetic double-hit" cases with $B C L 2$ and $C$ $M Y C$ rearrangements were not observed. 
Table 3 Immunohistochemical staining results

\begin{tabular}{|c|c|c|c|c|c|c|c|c|c|c|}
\hline & $\mathrm{COO}$ & $\mathrm{bcl} 2$ & c-myc & CD5 & CD95 & CD168 & Cyclin E & FOXP1 & pSTAT3 & EBER \\
\hline Evaluable cases & 123 & 123 & 123 & 123 & 123 & 123 & 123 & 123 & 123 & 123 \\
\hline Mean $\%$ of stained cells \pm SD & na & $34 \pm 38$ & $32 \pm 26$ & $2 \pm 14$ & $32 \pm 42$ & $4 \pm 9$ & $8 \pm 13$ & $31 \pm 38$ & $19 \pm 27$ & na \\
\hline N (\%) above cutoff & na & $34(28)$ & $44(36)$ & $4(3)$ & $60(48)$ & $38(31)$ & $30(24)$ & $44(36)$ & $44(36)$ & $2(1.5)$ \\
\hline Mean $\%$ of stained cells \pm SD in positive cases & na & $94 \pm 10$ & $61 \pm 20$ & $72 \pm 27$ & $65 \pm 38$ & $13 \pm 13$ & $26 \pm 13$ & $79 \pm 17$ & $49 \pm 26$ & $33 \pm 25$ \\
\hline Germinal center B cell (GCB) like, N (\%) & $42(34)$ & $11(32)^{a}$ & $16(36)^{a}$ & $1(25)^{a}$ & $22(37)^{\mathrm{a}}$ & $18(47)^{\mathrm{a}}$ & $11(37)^{\mathrm{a}}$ & $8(18)^{a}$ & $17(39)^{a}$ & $1(50)^{\mathrm{a}}$ \\
\hline
\end{tabular}

Except for FOXP1, which was of prognostic significance as an isolated marker, all other relevant proteins for cell of origin (COO) classification according to the Tally algorithm are summarized within the COO column na not applicable

${ }^{\mathrm{a}} \mathrm{GCB}$ out of the positive cases

The presence of $B C L 2$ breaks correlated with expression of bcl2 (rho $=0.355, p=0.001), \mathrm{CD} 10$ (rho $=0.388$, $p<0.005$ ), and GCB (rho $=0.302, p=0.006$ ). As expected, expression of GCET, bcl6, CD10, and LMO2 correlated with each other (GCB COO). pSTAT3 correlated with MUM1p, FOXP1, bcl6, and CD168 (rho = $0.301-0.473, p=0.01-0.0001)$. FOXP1 correlated with bcl2, MUM1p, and c-myc (rho $=0.429,0.438$, and 0.319, respectively, $p<0.001$ ). Expression of CD5 did not correlate with any of the examined single variables but showed a weak correlation with the so-called phenotypic $\mathrm{bcl} 2 / \mathrm{c}-$ myc double hits $(\mathrm{rho}=0.24, p=0.02)$. Phenotypic bcl2/c-myc double hits [34] correlated with expression of FOXP1 (rho $=0.379, p=0.0002$ ) and $B C L 2$ rearrangements (rho $=0.319, p=0.005$ ).

\section{Outcome analysis}

The primary study endpoint, i.e., EFS at 2 years, correlated with failure to achieve response according to international criteria and failure to achieve complete combined metabolic and morphologic response or metabolic response (rho values for all $>0.470, p$ values for all $<1 \mathrm{e}-5$ ). The median follow-up period was 53 months (95\% CI 45-51). There were 48 events, including 16 lethal events and 12 relapses 3 months after achievement of CR, of which 6 occurred $>12$ months after initial diagnosis. The 16 lethal events encompassed 9 deceases with/of disease and 7 deaths unrelated to cancer. Mean OS was 68 months $(95 \%$ CI 64-71), mean PFS was 59 months (95\% CI 53-65), and mean EFS was 46 months (95\% CI 40-52); median OS, PFS, and EFS for the whole collective were not reached.

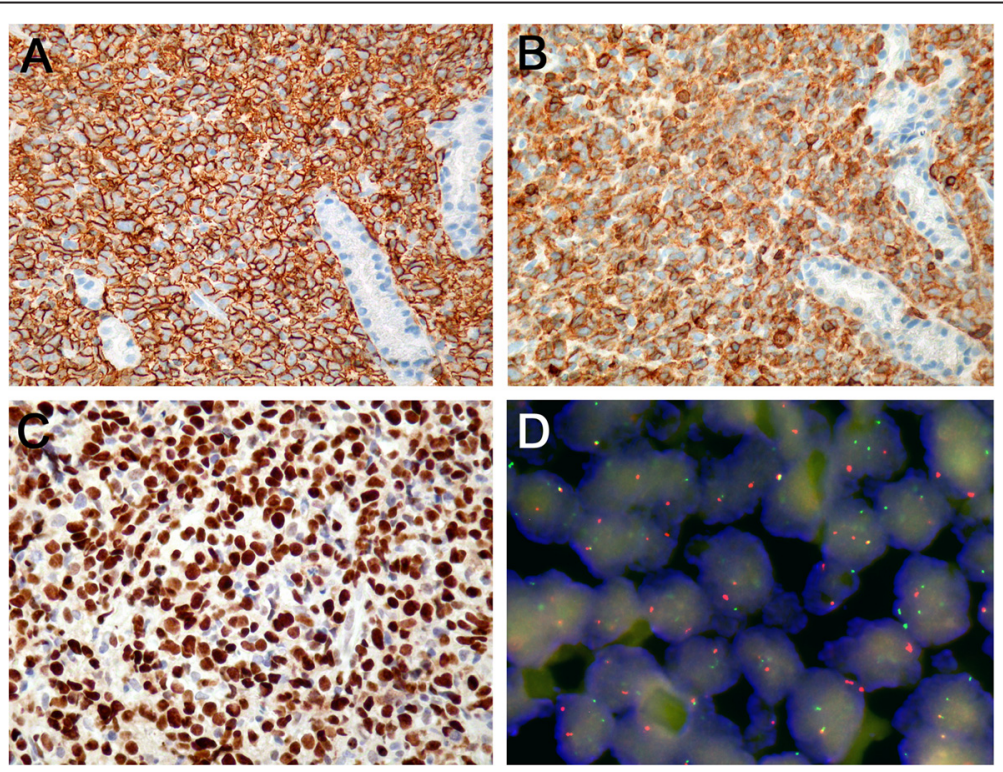

Fig. 1 Microphotographs of selected cases. Co-expression of CD20 (a) and CD5 (b) in an extranodal (intestinal) CD5-positive diffuse large B cell lymphoma. Microphotographs have been taken from consecutive sections; note deeper sections in $\mathbf{b}$ of the same glandular structures from $\mathbf{a}$. Original magnification $\times 320$. c Expression of FOXP1 in a positive case. Original magnification $\times 400$. $\mathbf{d}$ Split red and green signals corresponding to a $B C L 2$ gene rearrangement (translocation). Fused yellow signals corresponding to the non-translocated allele. Original magnification $\times 800$ 
All biomarkers were assessed for their prognostic importance after rational dichotomization (cutoffs listed in Table 1). Factors able to predict worse EFS in univariate Kaplan-Meier models were failure to achieve response according to international criteria, failure to achieve complete combined metabolic and morphologic response or metabolic response ( $p$ values for all $<0.005$ ), expression of CD5 ( $p=0.02$; Fig. 2a), and higher stage $(p=0.021)$. Factors predicting inferior PFS were failure to achieve response according to international criteria, failure to achieve complete combined metabolic and morphologic (but not only metabolic) response $(p<0.005)$, higher IPI $(p=0.006)$, higher stage $(p=0.005)$, presence of either $C-M Y C$ or $B C L 2$ gene rearrangements ( $p=0.033$; Fig. $2 \mathrm{~b})$, and expression of cyclin $\mathrm{E}$ in $>12 \%$ of tumor cells $(p=$ 0.046; Fig. 2c). Finally, factors predicting inferior OS were failure to achieve response according to international criteria, failure to achieve complete combined metabolic and morphologic (but not only metabolic) response ( $p$ values for all $<0.005)$, expression of FOXP1 in $>50 \%$ of tumor cells $(p<0.005$; Fig. $2 d)$, expression of cyclin E in $>12 \%$ of tumor cells $(p=0.005)$, expression of CD5 $(p=0.007)$, expression of $\mathrm{bcl} 2$ in $>70 \%$ of tumor cells $(p=0.016)$, expression of CD95 in any tumor cell $(p=0.018)$, and expression of pSTAT3 in $>17 \%$ of tumor cells $(p=0.025)$. All other clinico-pathological and phenotypic variables were not of prognostic significance respecting EFS, PFS, and OS. The multivariable analyses' results for EFS, PFS, and OS are shown in Table 4. Subgroup analysis limited to the DLBCL, not otherwise specified (NOS) cohort (omitting PMBL, THRBCL, and LG because of their more specific biology) revealed that expression of CD5 $(p=$ 0.044) retained its independent prognostic significance with respect to EFS (more sensitive for early events) and expression of FOXP1 $(p=0.004)$ with respect to OS (later events), while all other biomarkers failed to add prognostic information. In the case of CD5 because of the only weak correlation of CD5 with phenotypic bcl2/c-myc double hits, the limited number of CD5-positive cases, and the lacking prognostic significance of phenotypic bcl2/c-myc double hits in that series, multivariable analysis was not adjusted for phenotypic bcl2/c-myc double hits. Adjustment for phenotypic bcl2/c-myc double-hit scores in the case of FOXP1 showed that it retained its prognostic significance in those DLBCL, NOS cases scored 0 and 1 (and outperformed failure to achieve combined metabolic and morphologic remission in cases scored 0), but neither expression of FOXP1 nor failure to achieve complete combined metabolic and morphologic remission were of prognostic significance with respect to OS in phenotypic bcl2/c-myc double-hit score 2 DLBCL, NOS cases (data not shown in detail).

Since CD5 expression appeared to be of significant relevance, we thoroughly revised the four CD5-positive cases and evaluated multiple immunohistochemical markers to exclude blastoid mantle cell lymphomas (shown above). The four CD5-positive DLBCL were negative for cyclin D1 and SOX11 and expressed p27. These cases stained positively for CD5 in 50 to $100 \%$ of tumor cells did not show an intravascular component and were negative for
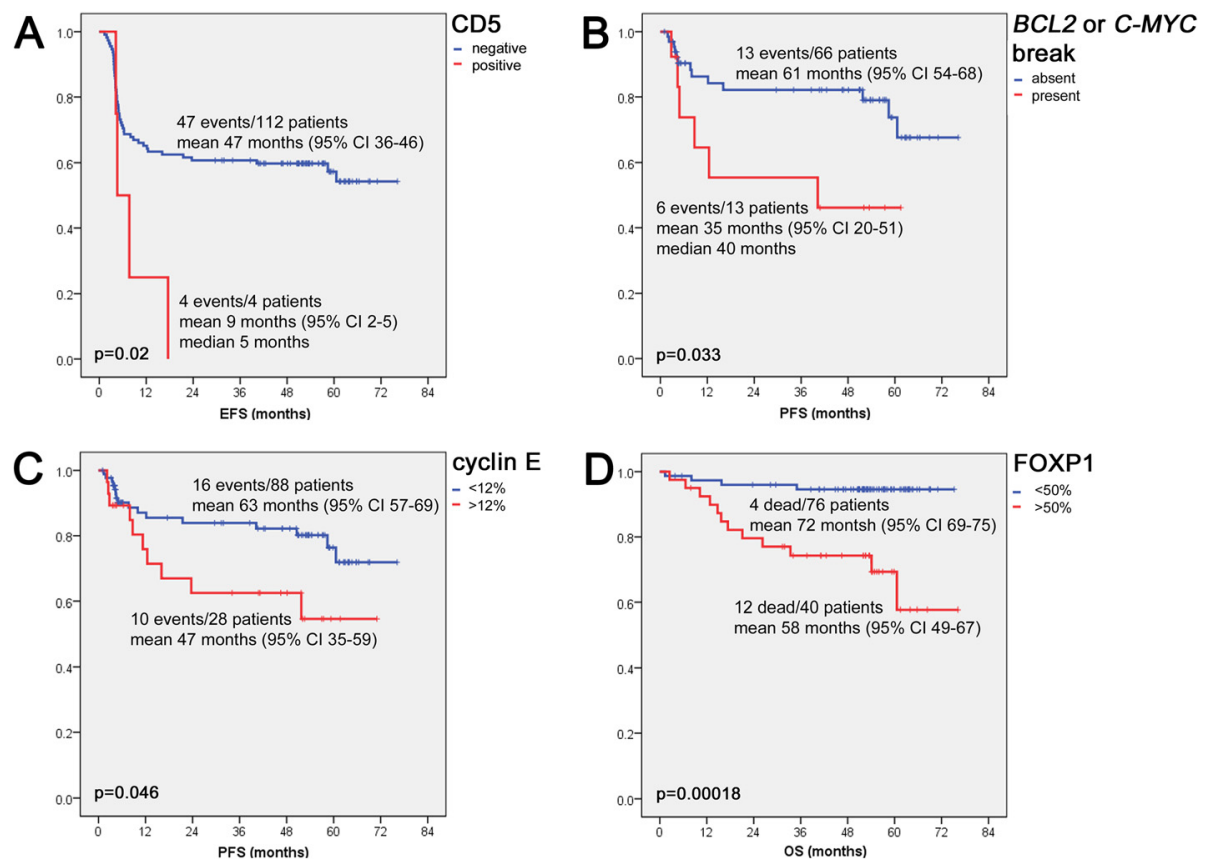

Fig. 2 Survival curves. Event-free (EFS) (a), progression-free (PFS) (b, c), and overall survival (OS) (d) with respect to biomarker expression 
Table 4 Multivariable analysis

\begin{tabular}{|c|c|c|c|c|}
\hline Survival & Parameter & Hazard ratio & $95 \% \mathrm{Cl}$ & $p$ value \\
\hline \multirow[t]{2}{*}{ Event-free } & Lack of complete combined metabolic and morphologic remission & 2.54 & $1.84-3.51$ & $<0.005$ \\
\hline & Expression of CD5 & 2.99 & $1.02-9.13$ & 0.047 \\
\hline Progression-free & Lack of complete remission according to international criteria & 16.39 & $4.57-58.82$ & $<0.005$ \\
\hline \multirow[t]{2}{*}{ Overall } & Expression of FOXP1 & 5.61 & $1.34-23.4$ & 0.018 \\
\hline & Lack of complete combined metabolic and morphologic remission & 1.93 & $1.04-3.58$ & 0.038 \\
\hline
\end{tabular}

Only significant results are shown

EBER; three were classified as non-GCB, while one was GCB; and three showed centroblastic morphology, while one was classified as centroblastic with increased immunoblasts. None of these four CD5-positive cases showed presence of either $C-M Y C$ or $B C L 2$ gene rearrangements; however, two patients fulfilled phenotypic criteria for double-hit lymphoma, expressing bcl 2 or c-myc above the respective cutoff scores. Two patients were male; two suffered from nodal lymphomas; two were Ann Arbor stage II, while the other two were stage I and III, respectively; and two patients had an IPI of 1 and two an IPI of 2 . The mean age of the CD5-positive patients was $64 \pm 13$ years, while that of the CD5-negative was $58 \pm 13$ (difference not of statistical significance). Two of the four patients failed to achieve remission (one of these two patients died of/ with lymphoma) and in the other two DLBCL relapsed after 8 and 38 months, respectively. Finally, DNA of the four CD5-positive cases was extracted and subjected to array comparative genomic hybridization (aCGH) analysis (Fig. 3) exactly as described elsewhere [35]. The analysis was successful in two cases and showed recurrent gains of $19 \mathrm{q}$ and losses of 1q43 [36], thus further corroborating the diagnosis of DLBCL. One of the cases showed specific loss of 9p21 (INK4A locus, also known as p16) known to be associated with DLBCL resistance to R-CHOP [37].

\section{Discussion}

Within this prospective study, we identified potential biomarkers (expression of CD5 for EFS and expression of FOXP1 for OS) that were able to predict the course of DLBCL at diagnosis, independent of stage and IPI. As expected ([38] and literature therein), dynamic parameters, such as response to therapy and especially failure to achieve complete remission, which are not obtainable at diagnosis, seem to be the most reliable outcome indicators in DLBCL, yet expression of CD5 and FOXP1 added information independent of these disease dynamic parameters.

Concerning the central aim of our study, i.e., to detect in situ biomarkers that reliably help predicting the outcome of DLBCL in a prospective, homogeneously treated collective of patients, our phenotypic and genotypic analyses show that carefully selected indicators such as CD5 might identify small yet prognostically relevant subgroups with adverse outcomes under R-CHOP. CD5 as biomarker has a special sensitivity towards early adverse events, which might not be the case for some of the currently propagated biomarkers of prognostic relevance such as c-myc expression/C-MYC gene status. Furthermore, our data reappraise the prognostic role of FOXP1 with respect to OS. Several other previously studied biomarkers with suspected prognostic potential
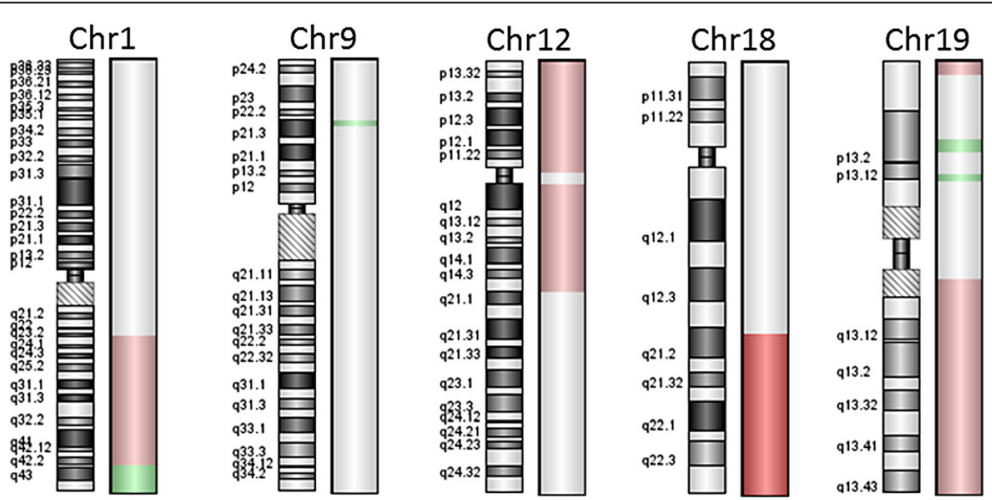

Fig. 3 Copy number aberrations in a CD5-positive case. Only aberrant chromosomes are shown. Green represents losses, and red represents gains of DNA. Darker red in the long arm of chromosome 18 indicates high-level amplification of this region. Note the small deletion at 9p21.3 (INK4A locus), known to be associated with chemoresistance 
like COO, expression of bcl2, or phenotypic double-hit score appeared to be less potent in the studied collective. This might in part be due to the small size of our study, in part to genuine properties of these markers, and in part to the fact that some of these markers, while being applicable to CHOP-treated DLBCL patients, are not applicable to cases treated with R-CHOP [39]. Considering our study size, there are obvious and inevitable limitations. Yet, because of the other characteristics of our collective (123 uniformly treated patients with a median follow-up period of 53 months and altogether 51 adverse events), our data solidifies understanding of the prognostic importance of in situ biomarkers in DLBLC and the 2-year EFS analysis delivers important results. Respecting the genuine properties of some markers, especially those used as surrogates to determine $\mathrm{COO}$, our results as well as observations of others [14] seriously challenge their reliability to identify prognostically and/or biologically meaningful groups among DLBCL.

Our observed prognostic role of CD5 and FOXP1 and possible prognostic role of bcl2 as well as structural genetic aberrations of (either) BCL2 or $C-M Y C$ are supported by other reports ([31, 40-46] and literature therein). While a considerable number of recent papers focused on the role of bcl2 and c-myc in DLBCL [34, 46, 47], it seems that CD5 merits special attention for several reasons: (a) it can be very easily detected in DLBCL by standard application of CD5 (instead of CD3) immunohistochemistry in the primary diagnostic panel with subsequent application of CD3 in CD5-positive cases (to subtract the "true" T cells), as well as CD23, cyclin D1, and SOX11 (to exclude transformed small lymphocytic B cell lymphomas and blastoid mantle cell lymphomas); (b) the respective cases express CD5 in a high proportion of tumor cells (>50-100 \%) with a moderate to strong staining intensity, and thus, its evaluation is unequivocal without the need for subjective and error-prone cutoff scores; and (c) because there is an increasing body of literature suggesting that CD5-positive DLBCL might represent a distinct biologic entity, being more prone to intravascular spread and extranodal location (particularly CNS), affecting individuals from the Far East and displaying a more aggressive behavior probably requiring alternative treatment approaches [40]. CD5-positive DLBCL are typically ABC [42, 48], show recurrent gains of $16 p$ and losses of $1 p$ and of 9q21 [36, 49], the latter being involved in chemoresistance [37], and display downregulation of extracellular matrix-related genes and upregulation of neurological function-related genes [48]. Addition of rituximab to $\mathrm{CHOP}$ improved the survival of CD5-positive DLBCL patients [50]; however, similarly to our results, the outcome of these patients is still significantly poorer compared to CD5-negative DLBCL patients [51], and the rate of CNS involvement seems not to be lowered by rituximab [52]. A recent very large retrospective report on 879 R-CHOP-treated DLBCL cases convincingly showed CD5 to be an IPI (and bcl2 and pSTAT3)-independent prognosticator in DLBCL as well [53] and pointed out distinct clinico-pathological peculiarities of such patients such as increased age, bone marrow spread, poor performance status, and B symptoms. Considering the possible direct biological effect of CD5 on B cells, namely its role as a negative regulator of $B$ cell signaling, its influence on the ERK, PI3K, and calcineurin pathways as well as survival stimulation through autocrine IL10-related loops and the predominant expression of integrin beta- 1 on the tumor cells, CD5 seems to be of probable functional and therapeutic importance for targeted approaches [40,54-56]. In addition, CD5-positive cases seem to overexpress bcl2, CARD11, CCND2, and FOXP1 at the protein and mRNA level and to be more rich in c-Rel, p65, and pSTAT3 [53], all known to identify DLBCL patients at risk; this study [53] also confirmed [48] downregulation of cellular adhesion genes in such instances. Taken together, previous data and our observations might justify a separation of CD5-positive DLBCL out of the group of DLBCL, NOS, as a distinct clinicopathological entity in need of R-CHOP treatment alternatives and, probably, CNS prophylaxis.

The prognostic role of FOXP1 in DLBCL was well established in the "pre-rituximab" era ([45] and references therein), while less attention has been paid to it in RCHOP-treated cases. Importantly, prognostically relevant $\mathrm{COO}$ algorithms pay special attention towards expression of FOXP1 to classify non-GCB-like DLBCL and >90\% concordance with GEP was only achievable by consideration of FOXP1 in these algorithms (e.g., [29, 44]). In line with these results, the recent report on the very poor prognosis of DLBCL reciprocally expressing the endocytic protein Huntingtin-interacting protein 1-related (HIP1R) and FOXP1 (the latter being a direct repressor of the HIP1R gene), i.e., FOXP1(hi)/HIP1R(lo) patients [57], and our prospective study findings suggest a more substantial relevance of FOXP1 in DLBCL. Importantly, FOXP1 belongs to the most reproducibly assessable markers in DLBCL as shown in an international inter- and intrainstitutional and inter- and intra-observer study [58], further calling for its regular evaluation.

Unexpectedly, a significant (33 \% for FISH and $50 \%$ for aCGH) dropout of cases for genotypic studies was noted. Detailed analysis of these cases revealed that pre-analytic conditions like inappropriate application of un-buffered formalin, fixation duration, surrounding temperature, and exact dehydration procedures were probably more relevant for lack of analytic success than the exact amount of examined tissue. Indeed, these failures were evenly distributed between core needle biopsies and lymphadenectomy specimens but were more commonly observed among tissues from a few centers. As expected, diagnostic tissue 
obtained by core needle biopsy procedures (usually 14$18 \mathrm{G}$ needles) was not arrayable and was rapidly exhausted for purposes of the study, precluding further analyses. Since cohorts of prospective clinical trials are characterized by meticulous documentation and uniform treatment of patients (the latter, if not uniform, can more substantially affect disease prognosis than many biomarkers), biomarker analyses should desirably be performed on cases collected within such studies. Therefore, the amount and the pre-analytical handling of tissue required for study inclusion must be considered also under the aspect of biomarker analyses. This particularly implies that physicians obtaining and handling the respective biopsies as well as the pathology laboratories must take responsibility for error-free and safe pre-analytic conduits, guaranteeing optimal tissue fixation and dehydration, which are indispensable for an accurate morphologic, phenotypic, and genetic analysis. For practical purposes, the protocol for probe handling from the laboratory, which provided probes with least dropout on molecular testing, is given in Additional file 1: Table S1.

\section{Conclusions}

In summary, distinct biomarkers like CD5 and FOXP1 are able to prognosticate DLBCL course at diagnosis, independent of stage and IPI and independent of initial therapy response. For the design of prospective DLBCL studies, issues like review of the slides by a central pathology, pre-analytic factors such as time to and time of fixation, choice of fixative, and dehydration as well as handling of biological entities and sub-entities in the spectrum of aggressive large B cell lymphomas should be properly discussed and promptly addressed.

\section{Additional file}

Additional file 1: Table S1. Summary of pre-analytics in the lab, submitting probes with least number of molecular testing dropouts.

\begin{abstract}
Abbreviations
ABC: Activated B cell; aCGH: Array comparative genomic hybridization; AUROC: Area under the ROC curve; BAP: Break-apart probe; CNS: Central nervous system; COO: Cell of origin; DFP: Double-fusion probe; DLBCL: Diffuse large B cell lymphoma; ECOG: Eastern Cooperative Oncology Group; EFS: Event-free survival; FISH: Fluorescence in situ hybridization; GCB: Germinal center B cell; G-CSF: Granulocyte colony-stimulating factor; GEP: Gene expression profiling; HIV: Human immunodeficiency virus; IPI: International Prognostic Index; LG: Lymphomatoid granulomatosis; MRI: Magnetic resonance imaging; NOS: Not otherwise specified; OS: Overall survival; PET: Positron emission tomography; PSF: Progression-free survival; PMBL: Primary mediastinal B cell lymphoma; R-CHOP: Rituximab, cyclophosphamide, hydroxydaunorubicin, vincristine, prednisone; R-IPI: Revised International Prognostic Index; ROC: Receiver operating characteristic; THRBCL: T cell- and histiocyte-rich B cell lymphomas; TMA: Tissue microarray.
\end{abstract}

\section{Competing interests}

The authors declare that they have no competing interests.

\section{Authors' contributions}

AT wrote the manuscript. AT, DK, CM, and SD designed the study. AT, NL, and SM performed immunohistochemical and FISH analyses. AT and DK performed statistical analyses. DJ performed aCGH analyses and formatted the manuscript. All authors read and approved the final manuscript.

\section{Acknowledgements}

This study was supported by the Oncosuisse grant OCS 02072-04-2007.

\section{Author details}

${ }^{1}$ Institute of Pathology, University Hospital Basel, Schoenbeinstrasse 40, CH-4031 Basel, Switzerland. '2 Swiss Group for Clinical Cancer Research (SAKK), Effingerstrasse 40, CD-3008 Bern, Switzerland. ${ }^{3}$ Division of Hematology and Oncology, Cantonal Hospital Aarau, Tellstrasse house Nr. 40, CH-5001 Aarau, Switzerland.

Received: 20 April 2015 Accepted: 5 June 2015

Published online: 14 June 2015

\section{References}

1. Stein H, Warnke RA, Chan WC, Jaffe ES. Diffuse large B-cell lymphoma, not otherwise specified. In: Swerdlow SH, Campo E, Harris NL, Jaffe ES, Pileri SA Stein $\mathrm{H}$, Thiele J, Vardiman JW, editors. WHO classification of tumours of haematopoietic and lymphoid tissues. 4th ed. Lyon: WHO Press; 2008. p. 233-7.

2. Cultrera JL, Dalia SM. Diffuse large B-cell lymphoma: current strategies and future directions. Cancer Control. 2012;19:204-13.

3. Nastoupil LJ, Rose AC, Flowers CR. Diffuse large B-cell lymphoma: current treatment approaches. Oncology. 2012;26:488-95.

4. Anonymous. A predictive model for aggressive non-Hodgkin's lymphoma. The International Non-Hodgkin's Lymphoma Prognostic Factors Project. N Engl J Med. 1993, 329:987-94.

5. Sehn LH, Berry B, Chhanabhai M, Fitzgerald C, Gill K, Hoskins P, et al. The revised International Prognostic Index (R-IPI) is a better predictor of outcome than the standard IPI for patients with diffuse large B-cell lymphoma treated with R-CHOP. Blood. 2007;109:1857-61.

6. Zhou Z, Sehn LH, Rademaker AW, Gordon LI, Lacasce AS, Crosby-Thompson A, et al. An enhanced International Prognostic Index (NCCN-IPI) for patients with diffuse large B-cell lymphoma treated in the rituximab era. Blood. 2014;123:837-42

7. Sehn LH. Paramount prognostic factors that guide therapeutic strategies in diffuse large B-cell lymphoma. Hematology Am Soc Hematol Educ Program. 2012;2012:402-9.

8. Said JW. Aggressive B-cell lymphomas: how many categories do we need? Mod Pathol. 2013;26 Suppl 1:S42-56.

9. Pasqualucci L. The genetic basis of diffuse large B-cell lymphoma. Curr Opin Hematol. 2013;20:336-44.

10. Alizadeh AA, Eisen MB, Davis RE, Ma C, Lossos IS, Rosenwald A, et al. Distinct types of diffuse large B-cell lymphoma identified by gene expression profiling. Nature. 2000:403:503-11.

11. Shipp MA, Ross KN, Tamayo P, Weng AP, Kutok JL, Aguiar RC, et al. Diffuse large B-cell lymphoma outcome prediction by gene-expression profiling and supervised machine learning. Nat Med. 2002;8:68-74.

12. Tzankov A, Zlobec I, Went P, Robl H, Hoeller S, Dirnhofer S. Prognostic immunophenotypic biomarker studies in diffuse large B cell lymphoma with special emphasis on rational determination of cut-off scores. Leuk Lymphoma. 2010;51:199-212

13. De Jong D, Rosenwald A, Chhanabhai M, Gaulard P, Klapper W, Lee A, et al. Immunohistochemical prognostic markers in diffuse large B-cell lymphoma: validation of tissue microarray as a prerequisite for broad clinical applications-a study from the Lunenburg Lymphoma Biomarker Consortium. J Clin Oncol. 2007;25:805-12.

14. Gutierrez-Garcia G, Cardesa-Salzmann T, Climent F, Gonzalez-Barca E, Mercadal $\mathrm{S}$, Mate $\mathrm{J} \mathrm{L}$, et al. Gene-expression profiling and not immunophenotypic algorithms predicts prognosis in patients with diffuse large B-cell lymphoma treated with immunochemotherapy. Blood. 2011;117:4836-43.

15. Winter JN, Weller EA, Horning SJ, Krajewska M, Variakojis D, Habermann TM, et al. Prognostic significance of $\mathrm{BCl}-6$ protein expression in $\mathrm{DLBCL}$ treated with $\mathrm{CHOP}$ or R-CHOP: a prospective correlative study. Blood. 2006;107:4207-13.

16. Hara T, Tsurumi H, Goto N, Kanemura N, Yoshikawa T, Kasahara S, et al. Serum soluble Fas level determines clinical outcome of patients with diffuse 
large B-cell lymphoma treated with CHOP and R-CHOP. J Cancer Res Clin Oncol. 2009;135:1421-8.

17. Shustik J, Han G, Farinha P, Johnson NA, Ben Neriah S, Connors JM, et al. Correlations between BCL6 rearrangement and outcome in patients with diffuse large B-cell lymphoma treated with CHOP or R-CHOP. Haematologica. 2010;95:96-101.

18. Evens AM, Sehn LH, Farinha P, Nelson BP, Raji A, Lu Y, et al. Hypoxia-inducible factor-1 \{alpha\} expression predicts superior survival in patients with diffuse large B-cell lymphoma treated with R-CHOP. J Clin Oncol. 2010;28:1017-24.

19. Ott G, Ziepert M, Klapper W, Horn H, Szczepanowski M, Bernd HW, et al. Immunoblastic morphology but not the immunohistochemical GCB/nonGCB classifier predicts outcome in diffuse large B-cell lymphoma in the RICOVER-60 trial of the DSHNHL. Blood. 2010;116:4916-25.

20. Tomita N, Sakai R, Fujisawa S, Fujimaki K, Taguchi J, Hashimoto C, et al. SIL index, comprising stage, soluble interleukin-2 receptor, and lactate dehydrogenase, is a useful prognostic predictor in diffuse large B-cell lymphoma. Cancer Sci. 2012;103:1518-23.

21. Perry AM, Cardesa-Salzmann TM, Meyer PN, Colomo L, Smith LM, Fu K, et al. A new biologic prognostic model based on immunohistochemistry predicts survival in patients with diffuse large B-cell lymphoma. Blood. 2012;120:2290-6.

22. Hong J, Park S, Park J, Jang SJ, Ahn HK, Sym SJ, et al. CD99 expression and newly diagnosed diffuse large B-cell lymphoma treated with rituximab-CHOP immunochemotherapy. Ann Hematol. 2012;91:1897-906.

23. Horn H, Ziepert M, Becher C, Barth TF, Bernd HW, Feller AC, et al. MYC status in concert with BCL2 and BCL6 expression predicts outcome in diffuse large B-cell lymphoma. Blood. 2013;121:2253-63.

24. Winter JN, Li S, Aurora V, Variakojis D, Nelson B, Krajewska M, et al. Expression of p21 protein predicts clinical outcome in DLBCL patients older than 60 years treated with R-CHOP but not CHOP: a prospective ECOG and Southwest Oncology Group correlative study on E4494. Clin Cancer Res. 2010;16:2435-42.

25. Meignan M, Gallamini A, Haioun C. Report on the first international workshop on interim-PET-scan in lymphoma. Leuk Lymphoma. 2009;50:1257-60.

26. Cheson BD, Pfistner B, Juweid ME, Gascoyne RD, Specht L, Horning SJ, et al. Revised response criteria for malignant lymphoma. J Clin Oncol. 2007;25:579-86.

27. PET scans in patients with diffuse large B-cell lymphoma receiving rituximab, cyclophosphamide, doxorubicin, vincristine, and prednisone. http://clinicaltrials.gov/show/NCT00544219.

28. Mamot C, Klingbiel D, Hitz F, Renner C, Pabst T, Driessen C, et al. Final results of a prospective evaluation of the predictive value of interim PET in patients with DLBCL treated with R-CHOP-14 (SAKK 38/07). J Clin Oncol. 2015, [in press].

29. Meyer PN, Fu K, Greiner TC, Smith LM, Delabie J, Gascoyne RD, et al. Immunohistochemical methods for predicting cell of origin and survival in patients with diffuse large B-cell lymphoma treated with rituximab. J Clin Oncol. 2011;29:200-7.

30. Visco C, Li Y, Xu-Monette ZY, Miranda RN, Green TM, Li Y, et al. Comprehensive gene expression profiling and immunohistochemical studies support application of immunophenotypic algorithm for molecular subtype classification in diffuse large B-cell lymphoma: a report from the International DLBCL Rituximab-CHOP Consortium. Leukemia. 2012;2103-2113.

31. Tzankov A, Xu-Monette ZY, Gerhard M, Visco C, Dirnhofer S, Gisin N, et al. Rearrangements of MYC gene facilitate risk stratification in diffuse large B-cell lymphoma patients treated with rituximab-CHOP. Mod Pathol. 2014;27:958-71.

32. McShane LM, Altman DG, Sauerbrei W, Taube SE, Gion M, Clark GM. REporting recommendations for tumour MARKer prognostic studies (REMARK). Eur J Cancer. 2005;41:1690-6.

33. Nagel S, Hirschmann P, Dirnhofer S, Gunthert U, Tzankov A. Coexpression of CD44 variant isoforms and receptor for hyaluronic acid-mediated motility (RHAMM, CD168) is an International Prognostic Index and C-MYC gene status-independent predictor of poor outcome in diffuse large B-cell lymphomas. Exp Hematol. 2010;38:38-45.

34. Green TM, Young KH, Visco C, Xu-Monette ZY, Orazi A, Go RS, et al. Immunohistochemical double-hit score is a strong predictor of outcome in patients with diffuse large B-cell lymphoma treated with rituximab plus cyclophosphamide, doxorubicin, vincristine, and prednisone. J Clin Oncol. 2012;30:3460-7.

35. Juskevicius D, Ruiz C, Dirnhofer S, Tzankov A. Clinical, morphologic, phenotypic, and genetic evidence of cyclin D1-positive diffuse large B-cell lymphomas with CYCLIN D1 gene rearrangements. Am J Surg Pathol. 2014;38:719-27.

36. Tagawa H, Tsuzuki S, Suzuki R, Karnan S, Ota A, Kameoka Y, et al. Genome-wide array-based comparative genomic hybridization of diffuse large B-cell lymphoma: comparison between CD5-positive and CD5-negative cases. Cancer Res. 2004;64:5948-55.

37. Kreisel F, Kulkarni S, Kerns RT, Hassan A, Deshmukh H, Nagarajan R, et al. High resolution array comparative genomic hybridization identifies copy number alterations in diffuse large B-cell lymphoma that predict response to immuno-chemotherapy. Cancer Genet. 2011;204:129-37.

38. Benjamin JE, Chen GL, Cao TM, Cao PD, Wong RM, Sheehan K, et al. Long-term follow-up of patients with diffuse large B-cell non-Hodgkin's lymphoma receiving purged autografts after induction failure. Bone Marrow Transpl. 2010;45:303-9.

39. Frei $E$, Visco C, Xu-Monette ZY, Dirnhofer S, Dybkaer K, Orazi A, et al. Addition of rituximab to chemotherapy overcomes the negative prognostic impact of cyclin E expression in diffuse large B-cell lymphoma. J Clin Pathol. 2013;66:956-61.

40. Jain P, Fayad LE, Rosenwald A, Young KH, O'Brien S. Recent advances in de novo CD5+ diffuse large B cell lymphoma. Am J Hematol. 2013;88:798-802.

41. Salles G, de Jong D, Xie W, Rosenwald A, Chhanabhai M, Gaulard P, et al. Prognostic significance of immunohistochemical biomarkers in diffuse large B-cell lymphoma: a study from the Lunenburg Lymphoma Biomarker Consortium. Blood. 2011;117:7070-8.

42. Yamaguchi M, Nakamura N, Suzuki R, Kagami Y, Okamoto M, Ichinohasama $\mathrm{R}$, et al. De novo CD5+ diffuse large B-cell lymphoma: results of a detailed clinicopathological review in 120 patients. Haematologica. 2008;93:1 195-202.

43. Ennishi D, Takeuchi $K$, Yokoyama M, Asai H, Mishima Y, Terui Y, et al. CD5 expression is potentially predictive of poor outcome among biomarkers in patients with diffuse large B-cell lymphoma receiving rituximab plus CHOP therapy. Ann Oncol. 2008;19:1921-6.

44. Visco C, Tzankov A, Xu-Monette ZY, Miranda RN, Tai YC, Li Y, et al. Patients with diffuse large B-cell lymphoma of germinal center origin with $B C L 2$ translocations have poor outcome, irrespective of MYC status: a report from an International DLBCL rituximab-CHOP Consortium Program Study. Haematologica. 2013;98:255-63.

45. Hoeller S, Schneider A, Haralambieva E, Dirnhofer S, Tzankov A. FOXP1 protein overexpression is associated with inferior outcome in nodal diffuse large B-cell lymphomas with non-germinal centre phenotype, independent of gains and structural aberrations at 3p14.1. Histopathology. 2010;57:73-80.

46. Johnson NA, Slack GW, Savage KJ, Connors JM, Ben-Neriah S, Rogic S, et al. Concurrent expression of MYC and BCL2 in diffuse large B-cell lymphoma treated with rituximab plus cyclophosphamide, doxorubicin, vincristine, and prednisone. J Clin Oncol. 2012;30:3452-9.

47. Hu S, Xu-Monette ZY, Tzankov A, Green T, Wu L, Balasubramanyam A, et al. $\mathrm{MYC} / \mathrm{BCL} 2$ protein coexpression contributes to the inferior survival of activated B-cell subtype of diffuse large B-cell lymphoma and demonstrates high-risk gene expression signatures: a report from The International DLBCL Rituximab-CHOP Consortium Program. Blood. 2013;121:4021-31. quiz 4250.

48. Suguro M, Tagawa H, Kagami Y, Okamoto M, Ohshima K, Shiku H, et al. Expression profiling analysis of the CD5+ diffuse large B-cell lymphoma subgroup: development of a CD5 signature. Cancer Sci. 2006;97:868-74.

49. Yoshioka T, Miura I, Kume M, Takahashi N, Okamoto M, Ichinohasama R, et al. Cytogenetic features of de novo CD5-positive diffuse large B-cell lymphoma: chromosome aberrations affecting 8p21 and 11q13 constitute major subgroups with different overall survival. Genes Chromosom Cancer. 2005:42:149-57.

50. Niitsu N, Okamoto M, Tamaru Jl, Yoshino T, Nakamura N, Nakamura S, et al. Clinicopathologic characteristics and treatment outcome of the addition of rituximab to chemotherapy for CD5-positive in comparison with CD5-negative diffuse large B-cell lymphoma. Ann Oncol. 2010;21:2069-74.

51. Hyo R, Tomita N, Takeuchi K, Aoshima T, Fujita A, Kuwabara H, et al. The therapeutic effect of rituximab on CD5-positive and CD5-negative diffuse large B-cell lymphoma. Hematol Oncol. 2010;28:27-32.

52. Miyazaki K, Yamaguchi M, Suzuki R, Kobayashi Y, Maeshima AM, Niitsu N, et al. CD5-positive diffuse large B-cell lymphoma: a retrospective study in 337 patients treated by chemotherapy with or without rituximab. Ann Oncol. 2011;22:1601-7.

53. Xu-Monette ZY, Tu M, Jabbar KJ, Cao X, Tzankov A, Visco C, et al. Clinical and biological significance of de novo CD5+ diffuse large B-cell lymphoma in Western countries. Oncotarget. 2015;6:5615-33.

54. Kobayashi T, Yamaguchi M, Kim S, Morikawa J, Ogawa S, Ueno S, et al. Microarray reveals differences in both tumors and vascular specific gene 
expression in de novo CD5+ and CD5- diffuse large B-cell lymphomas. Cancer Res. 2003;63:60-6.

55. Gary-Gouy H, Sainz-Perez A, Marteau JB, Marfaing-Koka A, Delic J, Merle-Beral $\mathrm{H}$, et al. Natural phosphorylation of CD5 in chronic lymphocytic leukemia B cells and analysis of CD5-regulated genes in a B cell line suggest a role for CD5 in malignant phenotype. J Immunol. 2007;179:4335-44.

56. Gary-Gouy H, Harriague J, Bismuth G, Platzer C, Schmitt C, Dalloul AH. Human CD5 promotes B-cell survival through stimulation of autocrine IL-10 production. Blood. 2002;100:4537-43.

57. Wong KK, Gascoyne DM, Brown PJ, Soilleux EJ, Snell C, Chen H, et al. Reciprocal expression of the endocytic protein HIP1R and its repressor FOXP1 predicts outcome in R-CHOP-treated diffuse large B-cell lymphoma patients. Leukemia. 2014:28:362-72.

58. Lawrie CH, Ballabio E, Soilleux E, Sington J, Hatton CS, Dirnhofer S, et al. Inter- and intra-observational variability in immunohistochemistry: a multicentre analysis of diffuse large B-cell lymphoma staining. Histopathology. 2012;61:18-25.

\section{Submit your next manuscript to BioMed Central and take full advantage of:}

- Convenient online submission

- Thorough peer review

- No space constraints or color figure charges

- Immediate publication on acceptance

- Inclusion in PubMed, CAS, Scopus and Google Scholar

- Research which is freely available for redistribution 